\title{
Freiheit in datafizierten Kontexten?
}

\section{Politische Betrachtung des digitalisierten Neoliberalismus}

\author{
Markus Baum
}

Angenommen: 1. April 2021 / Online publiziert: 26. April 2021

(C) Der/die Autor(en) 2021

Zusammenfassung Der Text verfolgt zwei Ziele: Ausgehend von Debatten zum Verhältnis von Digitalisierung und neoliberalem Kapitalismus geht er in einem ersten Schritt der Frage nach, inwiefern der hier als politische Philosophie verstandene Neoliberalismus und die digitale Mentalität der Solution eine Verbindung in Theorie und Praxis eingegangen sind. Dabei ist der Anspruch, bisher nicht berücksichtigte Widersprüche zwischen beiden freizulegen, um einer adäquaten politikwissenschaftlichen Diskussion des gegenwärtigen Digitalisierungsprozesses den Weg zu bereiten. In einem zweiten Schritt vollzieht der Text eine in der Tradition des Republikanismus stehende Kritik des Digitalisierungsprozesses neoliberaler Gesellschaften, dessen Kern als Datafizierung einer Vielzahl gesellschaftlicher Bereiche bestimmt wird. Da mit diesem begrifflichen Mittel systemisch angelegte Dynamisierungseffekte sowie eine spezifische Rationalität des Transformationsprozesses in den Blick gerückt werden, kann der Text insbesondere die freiheitsgefährende Unterminierung von Urteils- und Handlungspotentialen zur Diskussion stellen.

Schlüsselwörter Freiheit · Datafizierung · Digitalisierung · Neoliberalismus · Urteilen · Handeln

Dr. phil. M. Baum ( $\square)$

Katholische Hochschule Nordrhein-Westfalen, Robert-Schuman-Straße 25, 52066 Aachen, Deutschland

E-Mail: m.baum@katho-nrw.de 


\section{Freedom in a datafied environment?}

Political reflections of the digitalized neoliberalism

Abstract The article pursues two goals: Starting out from debates on the relationship between digitalization and neoliberal capitalism, he first examines to what extent neoliberalism, understood here as political philosophy, and the digital mentality of solutionism have merged in theory and practice. In order to prepare the way for an adequate discussion of the current digitization process in political science, the claim is to highlight contradictions between the two, which have not been taken into account so far. In a second step, the text criticizes the digitization process of neoliberal societies in the tradition of republicanism. The core of this process is defined as the datafication of a variety of social areas. In focusing its systemic dynamization effects as well its specific rationality, the text discusses the freedomthreatening erosion of judgement and action potentials.

Keywords Freedom · Datafication · Digitization · Neoliberalism · Judgement · Action

\section{Einleitung}

Der Digitalisierungsprozess wird im umfassenden Maße (arbeits- und wirtschafts-) soziologisch (Staab und Nachtwey 2016; Süssenguth 2015a) oder gesellschaftstheoretisch (Baecker 2018; Nassehi 2019) diskutiert. Oftmals wird jener Prozess in einem systematischen Zusammenhang mit der neoliberalen Wirtschaftsordnung betrachtet und auf den Begriff des digitalen Plattform-, Daten- oder Überwachungskapitalismus gebracht (Buckermann et al. 2017; Mayer-Schönberger und Ramge 2020; Seemann 2019; Staab und Nachtwey 2016; Zuboff 2018). Trotz der kaum vollständig zu überblickenden Forschungsliteratur lässt sich konstatieren, dass eine genuin politiktheoretische Reflexion des Zusammenhangs von Neoliberalismus und Digitalisierung ein Desiderat darstellt, dem sich jedoch in jüngster Zeit (zumindest mit Blick auf digitale Technologien) gewidmet wird (Berg und Thiel 2019; Berg et al. 2020; Jacob und Thiel 2017).

Ich möchte zur politikwissenschaftlichen Debatte beitragen, indem ich zwei Ziele verfolge: Zum einen gehe ich der Frage nach, inwiefern Neoliberalismus und Digitalisierung eine Verbindung eingegangen sind. Unzureichend beantwortet ist diese Frage bisher, da digitale Akkumulationskontexte kurzschlussartig neoliberal gedeutet werden, der Neoliberalismus somit als rein ökonomische Praxis missverstanden wird. Als eigenständige politische Philosophie wird er jedoch nicht begriffen. Im Digitalisierungsdiskurs führt diese Identifizierung von neoliberaler Praxis und Theorie dazu, dass die Inkongruenzen zwischen Neoliberalismus und der Mentalität, die dem Digitalisierungsprozess seine ideelle Form gibt und ihn legitimiert, systema- 
tisch zugunsten einer scheinbar widerspruchsfreien Symbiose ausgeblendet werden. ${ }^{1}$ Werden hingegen neoliberale Motive und Ideen als politische Philosophie ernstgenommen, treten sowohl die Gemeinsamkeiten also auch die Widersprüche hervor.

Zum anderen vollziehe ich eine in der Tradition des Republikanismus stehende Kritik des Digitalisierungsprozesses neoliberaler Gesellschaften, die den Fokus auf den widersprüchlichen Zusammenhang von digitalen Technologien, politischer Freiheit und politischem Handeln richtet. Auch hier weist das Forschungsfeld eine Leerstelle auf. So wird im gesellschaftswissenschaftlichen Kontext zwar der Einsatz digitaler Technologien im Kontext politischen Handelns evaluiert, jedoch werden diese lediglich als Mittel zum Zweck betrachtet, wie es Formulierungen über die „Nutzung digitaler Technologien“, die ,zur Verfügung stehen“, anzeigen (Züger et al. 2017, S. 265; Gerbaudo 2017). Im engeren Sinne politiktheoretische Ansätze reflektieren zwar den von Technologien gesetzten und zugleich begrenzten Handlungsrahmen mittels des Konzepts der Affordanz (so Berg et al. 2020), jedoch bringt dieses Konzept die Wirkmächtigkeit digitaler Technologien nicht umfassend zum Ausdruck. Der Fokus wird hier gerade von der ,strukturelle[n] Eigenlogik digitaler Technik“" (ebd., S. 178), die die republikanischen Ideen von Freiheit und politischem Handeln unterminiert, abgewendet und auf das Zusammenspiel von Affordanz und gesellschaftlichen Praktiken gerichtet. In einer explizit neorepublikanischen Perspektive diskutieren Hoye und Monaghan (2018) zwar die Möglichkeit einer Macht wider digitale Überwachung. Aufgrund ihres zentralen, Pettit entlehnten Konzepts der non-domination (Pettit 1997) tendieren sie jedoch zum einen dazu, das genuin republikanische Moment politischer Freiheit nicht zu berücksichtigen. ${ }^{2}$ Zum anderen unterlassen auch sie eine Auseinandersetzung mit der Eigenlogik digitaler Technologien, ein Desiderat, dass ebenfalls traditionell-republikanische Krisendiagnosen nicht füllen (Buchstein 1997; Sunstein 2001). Daher verfolge ich einen relationalen techniksoziologischen Ansatz, der zum einen die nicht-menschliche Handlungsfähigkeit berücksichtigt (maßgeblich dazu Latour 1996); und zum anderen Daten als Schnittstelle begreift, an der sich das Soziale mit dem Technischen verkoppelt (dazu Häußling 2020). Von Vorteil ist diese Betrachtung auch, insofern sie sensibel gegenüber spezifischen Rationalitäten ist, die sich im Digitalisierungsprozess bahnbrechen. So wird zwar in gegenwärtigen Debatten die Rationalität digitaler Mentalität als instrumentell charakterisiert und damit auf das Handeln und die Einstellungen von Individuen bezogen (Bock 2019; Houben 2018). Jedoch stellt insbesondere die Betrachtung der funktionalen Rationalität ein bisher vernachlässigtes, notwendiges Moment der politiktheoretischen Reflexion der Digitalisierung dar: Erst durch ihre Berücksichtigung können die systemisch angelegten Dynamisierungseffekte des Transformationsprozesses in den Blick geraten, die als Gefährdung von politischen

\footnotetext{
1 Der Mentalitätsbegriff wird hier im (wissens-)soziologischen Sinne verstanden, insofern er eine gruppenspezifische Art, die Welt zu interpretieren, zu evaluieren und zu legitimieren, meint. Der Begriff ist dabei durchaus offen dafür, dass eine Mentalität in gemeinsamen Erfahrungen im Umgang mit sozialen, politischen, ökonomischen oder eben technologischen Transformationsprozessen gründet (so Mau 2019, S. 244ff.).

2 So Richter (2018, S. 212-215) mit Blick auf Pettit.
} 
Freiheitspotentialen gelten müssen. ${ }^{3}$ Dazu ist es zudem notwendig, einen Digitalisierungsbegriff zu explizieren, der nicht allein den Einsatz digitaler Technologien, sondern darüber hinaus ihr gesellschaftstransformierendes Potenzial berücksichtigen kann. Dies leistet im vorliegenden Text der titelgebende Begriff der Datafizierung.

Um diese beiden Ziele zu verfolgen, rekonstruiere ich in einem ersten Schritt die zentralen Ideen und Motive des Neoliberalismus (2). In einem zweiten Schritt beleuchte ich sowohl die Mentalität, die den Digitalisierungsprozess auf ideeller Ebene prägt (3.1), als auch, wieso Datafizierung als Kern dieses Prozesses zu verstehen ist (3.2). Anschließend rücke ich Differenzen und Gemeinsamkeiten von Neoliberalismus und digitaler Mentalität in den Fokus (4.1). Im vorletzten Schritt folgt die politiktheoretische Diskussion der vorherigen Ausführungen unter besonderer Berücksichtigung der Frage, welche Konsequenzen sich für eine republikanisch verstandene Figur der Freiheit aus besagtem Prozess ergeben (4.2). Den Schluss bildet ein Ausblick auf eine Möglichkeit politischen Handelns in datafizierten Kontexte.

\section{Politische Philosophie und Praxis des Neoliberalismus}

Der Neoliberalismus wird derart häufig und mit Blick auf verschiedene Aspekte der Gesellschaft ins Zentrum von Analysen gerückt, ohne dass der Begriff hinreichend definiert wird und zur Debatte steht, ihn gar aus dem sozial- und politikwissenschaftlichen Vokabular zu streichen (Boas und Gans-Morse 2009; Hartmann 2018; Honneth 2016). Hier ist es hilfreich, zunächst zwischen den Ideen und Motiven des Neoliberalismus und ihren sozialen und ökonomischen Umsetzungen zu differenzieren, d.h. den Neoliberalismus als politische Philosophie zu begreifen (so jüngst Biebricher 2021).

Der Neoliberalismus formierte sich in den 1930er-Jahren als eine internationale Strömung in Deutschland, Österreich, England, Frankreich und den USA. Als dessen programmatische Schrift kann Lippmanns „An Inquiry into the Principles of the Good Society“ (Lippmann 1973), als deren zentrale Netzwerke das 1938 in Paris abgehaltene „Colloque Walter Lippmann“ sowie die 1947 gegründete „Mont Pèlerin Society“ verstanden werden (Butterwegge et al. 2016, S. 20f.). Zu dieser Strömung lassen sich die für den angloamerikanischen Raum prägende Wiener Schule um Hayek und die Chicago School um Friedman sowie die eher im deutschen und kontinentaleuropäischen Raum reüssierende Freiburger Schule zählen, zwischen denen ein wechselseitiger Austausch besteht. Die Wurzeln dieser Denktradition bilden sich in Opposition zum Nationalsozialismus, Faschismus und dem sowjetischen System sowie zur interventionistischen Makroökonomie Keynes' heraus. Dass sich jene politischen Systeme und wirtschaftspolitischen Leitbilder gleichen, ist die geteilte Annahme aller Neoliberalen, die im Ziel geeint sind, den Liberalismus zu reaktivieren, um auf besagte Feindbilder und die Krisentendenzen der damaligen Zeit zu reagie-

\footnotetext{
3 Das Motiv, zwischen instrumenteller und funktionalistischer Rationalität zu differenziere, verfolgt Habermas in seiner Theorie des kommunikativen Handelns (Habermas 1981b); ein grundlegender Zug dieser Theorie, der Houben (2018) bei seinem Versuch, mit Habermas den Digitalisierungsprozess zu kritisieren, irritierenderweise entgeht.
} 
ren. Mit der Veränderung der historischen Bedingungen, unter denen neoliberales Denken entstanden ist, wandelt sich ebenfalls der Neoliberalismus (Hayek 2003a, S. 36f.; Butterwegge et al. 2016, S. 14, 22). Gleichwohl lassen spezifische Motive und Theorieelemente exemplarisch als Kern des Neoliberalismus rekonstruieren, sodass dessen normative Vorstellungen über Gesellschaft, Individuum und Staat zutage treten. Insbesondere die Texte Hayeks bieten sich dazu an, geben sie doch den umfassendsten, aber auch schonungslosesten Einblick ins neoliberale Denken, der zugleich die radikale politische Dimension freilegt. ${ }^{4}$

Das normative Gravitationszentrum der Theorie Hayeks bildet die Idee negativer Freiheit, die als Abwesenheit derjenigen Grenzen verstanden wird, die verhindern könnten, „das [individuell; d. Verf.] Erstrebte zu erreichen“ (Hayek 1991, S. 58). Der Markt wird als konstitutiver Rahmen der neoliberalen Freiheitsidee betrachtet; denn, so die Argumentation, einzig der Markt gewährleistet eine ungezwungene Kooperation der Individuen, die nur dann mit anderen interagieren, wenn sie sich selbst einen Vorteil davon erhoffen. Freiheit heißt somit zudem, nur diejenigen Interaktionen einzugehen, die den eigenen Zwecken dienen (Friedman 1970, 2005, Kap. I). Den Rahmen jener Interaktionen bildet der Wettbewerb um knappe Ressourcen, der zugleich garantiert, dass die Individuen ihre Freiheit verantwortlich nutzen wesentlich für die Interaktionen ist somit nicht der Austausch, sondern die für jedes Individuum spürbare Konkurrenz (Foucault 2006, S. 171). Im Hintergrund dieser neoliberalen Idee steht die Annahme, dass Individuen Nutzenmaximierer*innen sind, wobei der verfolgte Nutzen der eigene, sprich kein gesellschaftlich oder politisch vorgegebener ist. Verantwortliches Handeln bedeutet demnach lediglich, aus Eigeninteresse seine Wettbewerbsposition zu verbessern und den individuellen Nutzen des Handelns zu erhöhen. ${ }^{5}$ Hingegen werden alle normativen Konzepte des Sozialen, zu denen der Neoliberalismus in fundamentaler Opposition steht, ebenso abgelehnt wie die utilitaristische Idee eines zwar strategisch verfolgten, jedoch kollektiv bestimmbaren Gesamtnutzens. Derartige Ideen führen neoliberalen Positionen zufolge zwangsläufig dazu, die darin enthaltenen Ideen sozialer Gerechtigkeit staatlich-autoritär durchzusetzen und das Individuum zu unterdrücken (Hayek 2003b, S. 70f., 117, 217, 126f.).

Ein weiteres neoliberales Argument gegen Formen sozialstaatlicher Politik gründet in der Annahme eines unhintergehbaren Nichtwissens. Insbesondere in zunehmend komplexen Gesellschaften kann es kein valides Wissen über die Gesellschaft und etwaige Handlungskonsequenzen geben (Hayek 1948, 1991, S. 30, 37 f.). Einzig der Wettbewerb wird als evolutionär fungierendes Ordnungsprinzip einer vernünftig eingerichteten, jedoch nicht aktiv geplanten Gesellschaft betrachtet (Hayek 2003b,

\footnotetext{
${ }^{4}$ Die Hervorhebung Hayeks gründet zum einen in der Methode des exemplarischen Vorgehens, das zugleich offen genug ist, weitere Vertreter des Neoliberalismus zu Wort kommen zu lassen. Dennoch wird der Neo- bzw. Ordoliberalismus der Freiburger Schule im vorliegenden Text weniger von mir berücksichtigt, da er zwar für die bundesrepublikanische Wirtschaftspolitik prägend (Ptak 2016), für die Verbindung von Digitalisierung und Kapitalismus jedoch zweitrangig ist. Zum anderen ragt Hayek innerhalb der neoliberalen Strömung hervor, insofern er als Führungsperson der „Mont Pèlerin Society“ gelten kann und seine Ideen die Chicagoer Schule maßgeblich beeinflussten.

${ }^{5}$ Die sozialen Facetten des Handelns werden im Kontext von Kleingruppen wie Familie oder Freundschaftskreise verortet (Hayek 1948, S. 29).
} 
S. 213). Denn er gilt als derjenige Mechanismus, der in der Lage ist, aus der Vielzahl einzelner Wissensbestände die angemessenen Gehalte zu selektieren. ${ }^{6}$ Aufgrund dieser Vorstellung optimaler, auf Konkurrenz basierender Ordnungsbildung sträubt sich der Neoliberalismus gegen alle Versuche der gestalterischen Planung, ganz gleich, ob sie von rechtsstaatlichen Demokratien oder totalitären Diktaturen initiiert wird. Planung muss als „Anmaßung von Wissen“ (so der Titel von Hayek 1975) begriffen werden, die zwangsläufig zu fehlerhaften Ordnungen führt (Hayek 1963).

In diesem Sinne ist jegliche neoliberale Bezugnahme auf Politik und staatliche Institutionen zunächst negativ, stellen sie doch die Grenzen der Freiheit dar. Ein zweiter, genauerer Blick zeigt jedoch, dass der Neoliberalismus spezifische Formen von Politik und Staatlichkeit ablehnt, andere wiederum befürwortet. Während ein umverteilender, den freien Markt rechtlich regulierender Sozialstaat, der Ideen sozialer Gerechtigkeit verfolgt und ggf. mit Zwang durchsetzt, kritisiert wird, wird ein Staat, der die institutionelle Ordnung des freien Wettbewerbs und des Privateigentums garantiert, ohne inhaltliche Ziele des Wettbewerbs (wie Vollbeschäftigung oder allgemeiner Wohlstand) zu definieren, durchaus willkommen geheißen (Foucault 2006, S. 244; Hayek 1994, S. 254; Lippmann 1945, S. 148 ff.). Trotz der Minimaldefinition darf die neoliberale Skizze keinesfalls als schwacher Staat missverstanden werden, im Gegenteil: Insbesondere im Ordoliberalismus wird er als starker Staat verstanden, der sehr potent konzipiert ist, um neoliberale Idee sukzessive gesamtgesellschaftlich durchzusetzen. Denn letztendlich wird freier Wettbewerb nicht als Naturzustand, sondern (gelegentlich nur insgeheim) als zu schaffende Ordnung verstanden (Brown 2019, S. 551, Fn. 22). „Der Neoliberalismus stellt sich also nicht unter das Zeichen des Laissez-faire, sondern im Gegenteil unter das Zeichen einer Wachsamkeit, einer Aktivität, einer permanenten Intervention“ (Foucault 2006, S. 188), um die Strukturen des freien Wettbewerbs garantieren zu können (Hayek 2003a, S. 37; auch Nozick 2006). Staat und Neoliberalismus sind daher als „ziemlich beste Feinde“ (Biebricher 2016) zu begreifen.

Nach dieser Darstellung tritt der politische Charakter des Neoliberalismus deutlich zu Tage. Der Versuch der Delegitimierung normativer Leitbilder wird vom genuin politischen Ziel grundiert, Gesellschaft aktiv zu gestalten, d.h. den Raum des Privaten auszuweiten, Kooperationszusammenhänge und soziale wie politische Institutionen zu privatisieren und so dem öffentlichen Zugriff zu entziehen (Hayek 1991, S. 169). Dabei ist eine spezifische Form der Rationalität für den Neoliberalismus auf zwei Ebenen charakteristisch. Auf der lebensweltlichen Ebene begreift er soziales Handeln einzig als strategische Interaktionsform, die sich am Maßstab ökonomischer Effizienz bemessen lässt. Diese strategische Rationalität zeigt sich zum anderen auf der Ebene wissenschaftlicher Theorien, die der Neoliberalismus als zu dominierendes Diskussionsfeld und entlang eines enorm ausgeprägten Freund-Feind-Schemas, in dem Gegen-Positionen als existentielle Gefahr betrachtet werden, interpretiert (Hayek 2003a, S. 270). Dementsprechend wird die eigene Wissensproduktion in erster Linie nicht mit Argumenten gegenüber anderen Positionen begründet, sondern lediglich durchgesetzt, um auf die Formierung von Gesellschaft Einfluss neh-

\footnotetext{
${ }^{6}$ Eine Ausnahme bildet hier Buchanans vertragstheoretischer Neoliberalismus, der durchaus eine vernunftgeleitete Gestaltung der Gesellschaft für möglich hält (Zinl 2016).
} 
men zu können. Zugespitzt formuliert, nehmen Neoliberale nur vordergründig eine diskursive, um Argumente bemühte Haltung ein und sind eigentlich primär an der Realisierung eigener Zwecke interessiert. ${ }^{7}$ Dieses Vorhaben ist „,total und universell“ (Butterwegge et al. 2016, S. 14) zugleich, da die gesamte Gesellschaft marktförmig organisiert werden soll und die (tendenziell verdeckt gehaltene) Normativität des neoliberalen Konzepts globale Gültigkeit beansprucht (Brown 2019, S. 545; affirmativ dazu Becker 1993). In diesem Sinne ist der Neoliberalismus als hegemoniales Projekt zu verstehen, das innerhalb gesellschaftlicher Kräfteverhältnisse situiert ist (Candeias 2009).

Es kann kaum bestritten werden, dass der Neoliberalismus eine enorm erfolgreiche politische Philosophie ist, die Gesellschaft(en) grundlegend transformiert (Brown 2015; Crouch 2011; zudem zur Praxis neoliberaler Transformation u.a. Bevir und McKee 2016; Peck 2016; Wissel 2016). Insbesondere ab den späten 1970er-Jahren dominieren neoliberale Ideen zunehmend ökonomische, soziale und politische Zusammenhänge. Man denke nur an Pinochets „Verfassung der Freiheit“, die es bereits Mitte der 1970er-Jahre der Chicago School um Friedman ermöglichte, das diktatorisch regierte Land als neoliberales Versuchsfeld zu nutzen. In der Phase einer weltweiten Rezession, die durch die zweite Ölpreiskrise ausgelöst wurde, können sich die in Chile praktisch erprobten neoliberalen Ideen als Antwort auf ökonomische Probleme präsentieren. Auf deren Grundlage werden spätestens ab den 1990er-Jahren in vielen anderen Ländern Arbeits- und Finanzmärkte dereguliert und liberalisiert, die Lebensverhältnisse flexibilisiert, öffentliche Leistungen und Institutionen privatisiert, der Sozialstaat abgebaut, eine kompromisslose Finanzpolitik der Austerität verfolgt, neue Absatzmärkte durch die Schaffung von Wettbewerb um verknappte Ressourcen generiert und entsprechende Formen einer Subjektivität motiviert (Bröckling 2007; Crouch 2008, Kap. 5; Dörre 2009; Münch 2011, Kap. 2; Voss und Weiss 2013). Der Internationale Währungsfonds, die Weltbank, Welthandelsorganisation und die G8-Gruppe, aber auch länderübergreifende Wirtschaftszusammenschlüsse wie NAFTA und EU tragen als internationale Organisationen und Institutionen dazu bei, neoliberale Ideen und Motive über den nationalstaatlichen Kontext hinaus zu realisieren. Auf diesem Wege wirken die theoretischen Voraussetzungen des Neoliberalismus daran mit, normativ gehaltvolle soziale Strukturen zu unterminieren. Obwohl in den 1990er-Jahren moderatere Formen der Regulierung und Aktivierung etabliert werden und das Verhältnis von Staat, Wirtschaft und Zivilgesellschaft neu vermessen wird, somit zivilgesellschaftliche Akteur*innen in Entscheidungsprozesse eingebunden werden (Mayntz 2004), wird zugleich das neoliberalen Kriterien folgende New Public Management innerhalb der Verwaltung implementiert (Häußermann et al. 2008, S. 295-298). Gegenwärtig spricht der französische Staatspräsident Emmanuel Macron gar davon, Frankreich zu einer Nation $\mathrm{zu}$ formen, die wie ein Start-Up denkt und handelt, und der (nun ehemalige) Leiter des White House Office, Jared Kushner, fordert, die US-amerikanische Regierung wie eine Firma zu führen (Brown 2019, S. 546).

Zusammengefasst kann der Neoliberalismus als eine politische Philosophie verstanden werden, innerhalb derer die Beziehung einer vollends marktförmig organi-

\footnotetext{
${ }^{7}$ Vgl. zu dieser Differenzierung Habermas (1981a, S. 126-151).
} 
sierten Gesellschaft und eines Staates, der diese Verfasstheit garantiert, modelhaft formuliert wird. In (wissenschafts-)politischer Perspektive erweist sich der Neoliberalismus als dezidierte Strategie, eine gesamtgesellschaftliche Hegemonie zu erlangen. Seine Praxis, die eigene Weltdeutung durchzusetzen, kann als erfolgreich gelten, insofern neoliberale Motive und Ideen eine Vielzahl gesellschaftlicher Bereiche konstituieren, die von Staaten und internationale Organisationen abgesichert werden. Mit dieser Charakterisierung der Theorie und Praxis des Neoliberalismus ist der Boden bereitet, auf dem er in Beziehung zu Mentalität und Prozess der Digitalisierung gesetzt werden kann.

\section{Mentalität und Prozess der Digitalisierung}

\subsection{Solutionism}

Um zu verstehen, in welcher Beziehung neoliberales Denken und die Mentalität, die einen spezifischen Einsatz technologischer Möglichkeiten skizziert und zugleich legitimiert, stehen, ist zunächst zu klären, wer überhaupt als Träger*in jener Mentalität gelten kann. Nachtwey und Seidl (2017) beantworten diese Frage, indem sie die von ihnen so bezeichneten digitalen Eliten in den Fokus rücken. Damit sind all jene Führungskräfte gemeint, die sich im und rund um das Silicon Valley ansiedeln und als Avantgarde gelten können, da sie einen gesellschaftlichen Wandel voranzutreiben suchen, indem sie ,programmatische Reden, Aktionärsbriefe, Ratgeberbücher und öffentliche Statements“ verfassen (Nachtwey und Seidl 2017, S. 12). Es sind u. a. herausragende Unternehmer*innen und Führungskräfte wie Mark Zuckerberg oder Nathan Blecharczyk sowie weitere CEOs und leitende Mitarbeiter*innen, die die beiden Autoren zu Wort kommen lassen und deren Selbstauskünfte sie nutzen, um im Anschluss an Boltanski und Chiapello (2003) eine „Polis der Solution“ (Nachtwey und Seidl 2017, S. 19-31) normativ zu rekonstruieren. Derart werden ideelle Motive und materielle Interessen, die hinter der Digitalisierung stehen, als ineinandergreifende Momente eines Weltbildes abgebildet, das in nicht unerheblichem Maß sowohl kapitalistische Wertschöpfungsketten als auch Gesellschaften insgesamt prägt (Nachtwey und Seidl 2017, S. 3, Fn. 3; Nachtwey und Staab 2015).

Die digitale Mentalität lässt sich als Reaktion auf die postfordistische Wirtschaftsformation, die von neoliberalen Ideen geformt wurde, verstehen. So kritisieren die digitalen Eliten eine nicht normativ eingehegte Akkumulation von Kapital. Insofern sie für ein ökologisches Bewusstsein und soziale Verantwortung plädieren, ziehen digitale Eliten eine normative Ebene in ihre wirtschaftliche Praxis ein, die vom Neoliberalismus gerade bekämpft wird. Durch die Aufnahme dieser Motive in das wirtschaftliche Handeln wird die Akkumulationsweise des Kapitalismus transformiert,

\footnotetext{
8 Nachtwey und Seidl (2017) erkennen darin einen Prozess, durch den der Kapitalismus auf eine breitere Legitimationsbasis gestellt wird. Eine derart normativ(istisch)e Rekonstruktion übersieht jedoch, so ließe sich antworten, die Momente von Gewalt und Zwang innerhalb des Kapitalismus, da lediglich normative Rechtfertigungsgrundlagen in den Fokus gerückt werden. Vgl. mit Blick auf moralisch-kognitivistische Verengungen der Rekonstruktion sozialer Transformationsprozesse Celikates (2019).
} 
ohne jedoch gänzlich verabschiedet zu werden. ${ }^{8}$ Denn die digitalen Eliten verstehe sich zwar als Erb*innen der sogenannten 68er-Bewegung; sie wollen jedoch soziale und ökologische Aspekte mit profitmaximierendem Wirtschaften verschmelzen. Entsprechend der Vorstellung einer Win-Win-Situation sind sie davon überzeugt, dass ihre sozialen und ökologischen Anliegen insbesondere als kapitalistisch erfolgreiche Ideen in dezentralen, flexiblen, spontanes Handeln ermöglichenden Strukturen durchsetzbar sind. Daher lassen sie sich als Idealtypus philanthropischer digitaler Unternehmer*innen verstehen (Barbrook und Cameron 1996; Turner 2009). Dass die Kopplung von Normativität und neoliberalem Kapitalismus durch den Einsatz smarter digitaler Technologien ermöglicht werden soll, ist Ausdruck eines Glaubens an wissenschaftliche Forschung, von Technikdeterminismus sowie der Haltung libertärer counter culture, in der Politik und demokratische Institutionen, staatliche Autorität und Bürokratie als Hemmnisse des weltverbessernden Perfektionismus gelten.

Das utopische Gesellschaftsmodell digitaler Eliten manifestiert sich im Bild einer technologisch vernetzen Gemeinschaft, in der alle Herausforderungen im Rahmen digitaler Geschäftsmodelle technisch gelöst werden (Nachtwey und Seidl 2017, S. 25, 30). Dieser Anspruch mag bereits überzogen wirken, jedoch reicht er noch weiter. Denn geleitet werden die Bestrebungen digitaler Eliten darüber hinaus von der Überzeugung, dass sich soziale Probleme und Krisen als technische Probleme umdeuten lassen, deren Lösung lediglich mathematischer Verfahren bedarf. Die (implizite) Annahme ist hier, die soziale Realität ließe sich in mathematischen Formeln abbilden und letztendlich vollständig berechnen. ,[R]egeln, was regelbar ist, und das nicht Regelbare regelbar machen“, so der Kybernetiker Schmidt (1941, S. 41), um die Gesellschaft durch Anpassung der technischen Möglichkeiten zu perfektionieren (Morozov 2014, 2016). In der Forschung wird dieses Weltbild auf den Begriff des „Solutionism“ (Morozov 2013) gebracht. Die Frage ist nun, wie genau der Strukturwandel charakterisiert werden kann, der durch die hier skizzierte Mentalität vorangetrieben wird.

\subsection{Digitalisierte und datafizierte Gesellschaft}

Die Basis der Digitalisierung sind Daten, d.h. distinkte Einheiten, die miteinander vernetzt werden können und müssen, um Informationen, Wissen oder Bedeutung zu generieren. Daten treten somit zunächst aggregiert in Form von Small Data auf, sprich als Datenbestände, die für die menschliche Kognition überschaubar bleiben. Um Datenbestände zu generieren, ist es notwendig, empirische Beobachtungen, auf die die Daten verweisen, methodisch kontrolliert in ein numerisches Relativ zu überführen (Schnell et al. 2005, S. 7-14). Getragen wird das Vorgehen, soziale Zusammenhänge in numerische Ordnungen zu überführen, von verschiedenen Annahmen: Daten seien eine universelle, nicht von Kultur oder Subjektivität verzerrte Sprache der Erkenntnis, die die Empirie objektiv abbildet (Bächle 2016, S. 111-125). Small Data können zu Big Data, sprich exorbitanten, von der menschlichen Kognition nicht mehr verarbeitbaren Datenmengen aggregiert werden, zu deren Erfassung es enorm leistungsfähiger Computer bedarf (Helbing 2015, S. 76f.). Durch die Etablierung der Kybernetik Mitte des 20. Jahrhunderts sowie die zunehmende Leistung von Hoch- 
leistungsrechnern seit der letzten Jahrtausendwende besteht die Möglichkeit, Big Data mittels statistischer Modelle und Verfahren der Mustererkennung auszuwerten (Kitchin 2014). Die erkannten Muster und Regelmäßigkeiten beruhen wiederum auf Clusterverfahren, in denen mehrdimensionale Zuordnungen von Merkmalen und Entitäten unterschiedlicher Granularität vorgenommen werden (Passoth und Rammert 2019, S. 163). Besagte Verfahren und Modelle nutzen die erstmals in den 1920er-Jahren auf Schaltkreisen implementierten Algorithmen. Algorithmen stellen eine „Formalisierung von Rechenvorgängen“ (Mersch 2019, S. 852) dar, die in der artifiziellen Symbolsprache der Mathematik abgebildet werden. Ausgehend von einer Dateneingabe und vorgegebenen, sozial und kulturell geprägten Verrechnungsregeln, ver- und bearbeiten Algorithmen Big Data im Rahmen automatisierter Prozesse (Aamodt und Nygård 1995). Determiniert sind diese Operationen, insofern Verrechnungsregeln und -reihenfolgen exakt vorgegeben sind. Im Unterschied zum mathematischen Denken setzt das algorithmische Prozessieren nicht allein zwingende logische Schlüsse, sondern eine stringente Prozessrichtung voraus, in der verschiedenen Systemzustände zeitlich geordnet werden. ${ }^{9}$

Die Differenz von Small Data und Big Data ist jedoch nicht rein quantitativer Art, da mit der Auswertung von Big Data weitere Hoffnungen auf Erkenntnis einhergehen. Durch den Modus der Aggregation sollen Big Data und durch diese vermittelt ein höheres Wissen generiert werden, das in kleineren Datenbeständen nicht vorliegt (Mayer-Schönberger und Cukier 2013, S. 76). Ein Blick in die jüngste Geschichte zeigt, dass in der Politik, der Arbeitswelt, der Wirtschaft und der Wissenschaft die Generierung von Big Data und die Anwendung von Algorithmen u.a. aufgrund dieser Hoffnung enorm zugenommen haben (Borgwardt 2018; Prietl und Houben 2018; Reichert 2014; Süssenguth 2015b). Die je eigenen Bereiche werden datenmäßig repräsentiert, in die formalisierte Symbolsprache der Mathematik überführt, um die nun qualitätslosen Einheiten innerhalb funktionaler Zusammenhänge darstellen zu können (Mayer-Schönberger und Cukier 2013, S. 78-83). Ein derartiges Vorgehen wird dazu genutzt, Verhaltensmuster abzubilden, Diagnosen zu erstellen, Wahlverhalten vorherzusagen oder Produktionsprozesse zu optimieren. Obwohl die Anstrengung, statistisch auswertbare Daten zu generieren, mit der Bildung von Nationalstaaten, der Etablierung der Sozialwissenschaft und rational verfahrender Bürokratie zu einem flächendeckenden Projekt wird (Spittler 1980; Vormbusch 2012), unterscheidet sich die digitalisierte Gesellschaft von nicht-digitalen Gesellschaften qualitativ dadurch, dass die Selbstbeobachtungen und -beschreibungen ersterer konstitutiv digitale Technologien nutzen, mittels derer das Soziale in binärer, Komplexität reduzierender, archivierbarer, mehrfach vernetz- und kombinierbarer Form erfasst wird (Baecker 2018).

Nun bleibt jedoch der Kern des gegenwärtigen Transformationsprozesses unberücksichtigt, wird die digitale Selbstbeschreibung von Gesellschaft(en) lediglich als digitale Repräsentation der sozialen Realität charakterisiert. Angemessener wird er

\footnotetext{
${ }^{9}$ Die Differenz von mathematischen Modellen und computertechnischem Denken zeigt sich darin, dass das ,=“-Zeichen durch das , $\Rightarrow$ "-Zeichen ersetzt wird, sodass aufgrund der Gerichtetheit des Prozesses eine Umkehrung, wie sie in der Algebra möglich ist, ausgeschlossen wird (Knuth und Pardo 1978, S. 206; Rutishauser 1956, S. 28).
} 
mittels des Begriffs der Datafizierung gefasst: Während Digitalisierung die Transformation von Inhalten in eine artifizielle Symbolsprache meint, bedeutet Datafizierung, dass die digitalen Daten in die sozialen Wirklichkeiten einfließen, sich mit sozialen Prozessen vernetzen, ,,sodass diese Wirklichkeiten eine grundlegende Wandlung erfahren“ (Häußling et al. 2017, S. 2). Denn da die von algorithmischen Rechenoperationen produzierten Resultate als Bestandteil des kulturellen Wissens verstanden werden können, arbeiten sie ebenfalls an der Konstitution sozialer Realität und erlangen auf diesem Wege die Kraft, Verbindlichkeiten im Umgang mit dieser zu generieren (Barlösius 2001, S. 183). ${ }^{10}$ Da im Zuge der Datafizierung das Soziale binär codiert wird und digitale algorithmische Prozesse wiederum binären Codes folgen, sind Daten als Schnittstellen zu verstehen, an denen soziale und technische Prozesse gekoppelt und für einander anschlussfähig gestaltet werden (Häußling 2020). Datafizierung bedeutet somit nicht allein ein stetiges Anwachsen des gesellschaftlichen Datenvolumens, sondern einen qualitativen Wandel der Konstitutionsprozesse sozialer Wirklichkeit. ${ }^{11}$

Innerhalb datafizierter Zusammenhänge amalgamieren sich zwei Arten des Denkens und Wahrnehmens, in deren Perspektiven Handlungs- und Beziehungsformen eigentlich verschieden aufgefasst werden: die instrumentelle und die strategische Rationalität. Instrumentelle Rationalität lässt die soziale Realität lediglich in empirisch nachweisbaren Regelmäßigkeiten von Prozessen und Strukturen, das heißt analog zu physikalischen Vorgängen hervortreten. Die Perspektive instrumenteller Rationalität ist nicht die von (am sozialen Geschehen) Teilnehmenden, sondern die von Beobachtenden (Mersch 2019). Zugleich werden Individuen im Zuge der Datafizierung sozialer Zusammenhänge zu Adressat*innen strategischer Manipulation und Steuerung (bspw. beim predictive policing oder nudging). Während instrumentelle Rationalität die soziale Realität objekthaft abbildet, begreift die strategische Rationalität Individuen zwar als Subjekte mit reflexiven Fähigkeiten. In konkreten

\footnotetext{
${ }_{10}$ Zur Illustration: Das EU-Forschungsprojekt INDECT (Intelligent information system supporting observation, searching and detection for security of citizens in urban environment), das von 2009 bis 2014 lief, widmete sich der Entwicklung intelligenter Sicherheitssysteme für das sogenannte predictive policing. Diese Systeme wurden als Schnittstelle konzipiert, um Daten aus öffentlich zugänglichen Internetprofilen, aus Überwachungskameras und aus Polizeidatenbanken zu verknüpfen. Kriminelles Verhalten soll so statistisch vorhergesagt werden, um anschließend sicherheitsspezifische Routinen anzustoßen (Bismarck 2019). Das mag nach ferner Zukunft klingen, ist jedoch gelebte Praxis in der alltäglichen Polizeiarbeit der Städte Kaliforniens. Dort werden Algorithmen und Big Data genutzt, um Verhaltensmuster abzubilden und Vorhersagen über strafrechtlich relevante Handlungen tätigen zu können. Die in den Fokus geratenen Personen werden anschließend auf eine Liste potenzieller Straftäter*innen gesetzt. Eine verstärkte Beobachtung oder vorsorgliche Hausbesuche, sprich stigmatisierende Maßnahmen durch Polizeibeamt*innen können die Folge sein (Kurz 2017; Zedner 2007).

${ }^{11}$ In diesem Sinn greifen die Gesellschaftstheorien von Nassehi (2019) und Baecker (2018) zu kurz, begreifen sie die Digitalisierung lediglich als Verdopplung der „Welt in Datenform“ (Nassehi 2019, S. 33f.). Da insbesondere Nassehi Digitalisierung als komplexitätsreduzierende Antwort auf die Moderne versteht, stellt dieser Prozess nur ein weiteres binär codiertes System innerhalb funktional differenzierter Gesellschaften dar (Nassehi 2019, S. 177). Diese Position läuft darauf hinaus, dass moderne Gesellschaften immer schon digital waren, der qualitative Unterschied, den Datafizierung hervorbringt, jedoch aus dem Blick gerät. Demgegenüber zeigt der Begriff der Datafizierung auf, dass der Digitalisierungsprozess quer zur funktionalen Differenzierung läuft, da er alle sozialen Systeme transformiert (so auch Lindemann 2020, S. 307).
} 
Interaktionen werden diese Fähigkeiten selbst jedoch nicht anerkannt, da das Ziel der Interaktion in der Beeinflussung dieser Subjekte und nicht im gemeinsamen sozialen Handeln besteht (dazu Habermas 2009, S. 39-42, 47 f.).

Unabhängig davon, ob Individuen als Objekt oder beeinflussbares Subjekt wahrgenommen werden, beziehen sich instrumentelle und strategische Rationalität auf die zwar einseitige, aber dennoch bewusste Gestaltung von Interaktionszusammenhängen. Beide Rationalitätsformen stehen somit in einem begrifflichen Zusammenhang mit Intentionalität und menschlicher Handlung. Die Rationalitätsformen der Datafizierung einzig derart zu rekonstruieren, greift jedoch zu kurz. Denn die datafizierenden Technologien entfalten in der spezifischen Form ihrer soziokulturellen Einbettung eine eigenständige Wirkung. Diese Wirkung wird in einer systemfunktionalistischen Perspektive in besonderer Weise beschreibbar. ${ }^{12}$ Datafizierung wird so als Prozess rekonstruierbar, der ein soziokulturelles und -technisches System etabliert, das einer eigenen Logik und dem Ziel der Systemstabilisierung folgt. Innerhalb dieses Systems fungieren zum einen Daten als Steuerungsmedien der Interaktionszusammenhänge, zum anderen Algorithmen als Koordinatoren des Handelns der Individuen - die über Daten und Algorithmen generierten Informationen konstituieren die Handlungsoptionen. Die funktionalistische Rationalität der Datafizierung besteht demnach nicht primär darin, Zwecke von Handlungen zu realisieren. Vielmehr werden sie an dem Ziel der Systemintegration ausgerichtet. Das heißt, (nicht zwingend intendierte) Handlungseffekte werden derart funktional vernetzt, dass die Verfolgung individueller Zwecke mittels datafizierender Technologien der Erhaltung des DatenSystems dienlich ist: Mögen noch so viele Einzelne ihre je eigenen Zwecke folgen, so werden ihre Handlungen dennoch nicht über den geteilten Zweck, sondern hinter ihrem Rücken durch Algorithmen und über das systemische Steuerungsmedium der Daten vernetzt, deren stetige Akkumulation den Datafizierungsprozess vorantreibt. Die funktionale Integration von Handlung in den Prozess der Datafizierung verstärkt jedoch nicht allein die Prozessdynamik, sondern bekräftigt so die Prämissen ihrer Anwendung. Denn intensive Nutzung datafizierender Technologien zeigt das Dargestellte im Medium der Daten. Da Medien und Technologien mehr als lediglich Trägerinnen von Informationen und Funktionen sind, sondern darüber hinaus weltbildende Wirkungen aufweisen, prägen sie das Bewusstsein derer, die sie nutzen. ${ }^{13}$ Die Auffassung, jegliche Aspekte der objektiven, sozialen und subjektiven Welt in datenform abbilden zu können, ist bis in die Gegenwart hinein derart hegemonial geworden, dass in der Wissenschaft gar von einem algorithmic turn und der ,datafication of everything“" gesprochen wird (Mayer-Schönberger und Cukier 2013, S. 94; zudem Mämecke et al. 2018; Uricchio 2011).

An dieser Stelle wird ersichtlich, inwiefern der Datafizierungsbegriff dem der Digitalisierung vorzuziehen ist, soll eine umfassendere Perspektive eingenommen werden. Denn erstgenannter ist sowohl enger und exakter gefasst, insofern er genau auf den soeben beschriebenen Kopplungsprozess bezogen ist; als auch weiter gefasst, bezieht er sich doch nicht allein auf die Nutzung und Vernetzung digitaler

\footnotetext{
12 Vgl. zu den folgenden Argumenten Habermas' (1981b, S. 226) Darstellung der Systemtheorie Parsons.

13 Vgl. für die Wirkung von (in diesem Falle Massenproduktions-)Technologien auf gesellschaftliche Wahrnehmungsmuster Benjamin 1991, S. 471-508.
} 
Kommunikationsgeräte und -plattformen, sondern auf gesellschaftliche Konstitutionsprozesse insgesamt. Zudem reflektiert der Datafizierungsbegriff die agency von nicht-menschlichen Akteuren, indem er Daten und Algorithmen als eigenständige Faktoren hervorhebt. So ermöglicht er, nicht allein die Frage zu verfolgen, wie digitale Infrastrukturen beschaffen sein sollten. Darüber hinaus sensibilisiert er dafür, welche gesellschaftlichen Strukturen gerade in einer politiktheoretischen Perspektive als unverfügbar für Datafizierung gelten müssen, sollen Potenziale des Politischen nicht unterminiert werden, eine Perspektive, die in der gegenwärtigen Debatte allzu oft außen vor bleibt.

Von hier aus lassen sich nun Gemeinsamkeiten und Widersprüche zwischen Neoliberalismus und digitaler Mentalität deutlich hervorheben und dann in der politiktheoretischen Perspektive des Republikanismus kritisch erörtern.

\section{Politische Kritik der datafizierten neoliberalen Gesellschaft}

\subsection{Digital und neoliberal?}

Um die marktförmige Umgestaltung aller gesellschaftlichen Bereiche zu optimieren, müssen Informationen über die jeweiligen Interaktionszusammenhänge zur Verfügung stehen, damit diese nach Kriterien der Effizienz und Effektivität beurteilt werden können; und diese Informationen müssen ebenso effizient und effektiv verarbeitet werden können, um die Interaktionen im Bedarfsfall anzupassen (Castells 2000; Harvey 2011, S. 3; Terranova 2004). Allein hier erweist sich die Datafizierung sozialer Zusammenhänge zumindest als hilfreiche Technologie zur Beschaffung von Informationen, insofern sie Möglichkeiten eröffnet, (eine nun exorbitante Menge von) Daten über Kund*innen oder Wähler*innen etc. zu sammeln und passgenaue Konsum-/Politik-Angebote usw. zu adressieren (Schaupp 2017; Schaupp und Jochum 2019, S. 336 ff.). Eine weitere Wahlverwandtschaft gründet in den Bewertungsschemata der neoliberalen Gesellschaft. Diese basieren auf numerischer Logik, sind also datenbasiert: Noten, Evaluationen, Rankings werden gelegentlich zwar qualitativ beschrieben (sehr gut, gut, mit Auszeichnung, ausreichend etc.); im Hintergrund stehen jedoch metrische Skalen, die die sozialen Bereiche quantifizieren und so zugleich Vergleichbarkeit und Hierarchien generieren. In dieser Perspektive erweist sich Datafizierung als funktionales Element einer neoliberalen Gesellschaft, ermöglicht sie doch, die soziale Realität in eine quantitativ-metrische Ordnung zu übersetzen und Objektivierungen, Rangbildungen sowie eine datenbasierte (Selbst-)Kontrolle (noch weiter) voranzutreiben (Boyd und Crawford 2013; Hutchby 2016; Mau 2017, S. 17f., 27, 40-47 sowie Kap. 3; Schaupp und Jochum 2019). Im Bannkreis gegenwärtiger sozialer Transformationsprozesse verschmilzt „der neoliberale Geist mit einem kybernetischen Steuerungswissen“ (Nosthoff und Maschewski 2017, online).

Mit dieser Verschmelzung geht ein Verlust von Freiheitspotentialen einher. Denn der Neoliberalismus bedeutet letztendlich nicht, Institutionen und Individuen in einen unkontrollierten Wettkampf aller gegen alle zu entlassen; Kontrolle wird in neoliberalen Gesellschaften subtil(er) ausgeübt, indem sozialen Verhältnisse derart 
restrukturiert werden, dass sich Individuen und Institutionen als eigenständige und eigenverantwortliche, in Konkurrenz agierende Akteur*innen begreifen. In seiner Analytik der Macht bringt Foucault diese Form der Subjektivierung auf den Begriff der Gouvernementalität, in der Autonomie und Heteronomie konstitutiv ineinander greifen (Foucault 2005, S. 687-707, 2006). ${ }^{14}$ Bleibt innerhalb der neoliberalen Transformation der Gesellschaft somit ein Raum gouvernementaler Freiheit erhalten, da Momente der Unterwerfung und Ermächtigung innerhalb der sozialen Verhältnisse ineinandergreifen, so versiegt diese mit der Implementierung datafizierender Steuerungsmodelle in neoliberale Strukturen. Denn die Institutionalisierung funktionalistischer Rationalität bringt ein sich selbst integrierendes System hervor, in dem Spontanität und jene Freiheit zum einen von den konstitutiven Systemprozessen, zum anderen von einem objektivistisch-szientistischen Weltbild vollends verdrängt werden, sodass Individuen einzig als manipulier- und steuerbar begriffen und behandelt werden. Hier zeigen sich weitere Reibungen zwischen Neoliberalismus und digitaler Mentalität. Denn die Steuerungsmöglichkeiten, die die datafizierenden Technologien eröffnen, stehen in Widerspruch zur ungeplanten Interaktion, die die politische Philosophie des Neoliberalismus normativ ausweist, und konterkarieren das Idealbild eines freien Marktes (Schaupp und Jochum 2019, S. 330 ff.). Auf die geteilte Annahme eines konstitutiven Nicht-Wissens wird somit von beiden unterschiedlich reagiert: Der Neoliberalismus baut auf die konkurrenzgetriebene egoistische Interaktion und die spontane Ordnungsbildung; die digitale Mentalität stellt deren Potenzial grundlegend infrage und vertraut hingegen Big Data und Algorithmen. Der Frage, wie sich dieser Zusammenhang politisch reflektieren lässt, wird im Folgenden nachgegangen.

\subsection{Freiheit und politisches Handeln - und datafizierte Strukturen}

Insbesondere das republikanische Denken Arendts eignet sich, den Datafizierungsprozess in politiktheoretischer zu diskutieren, ermöglicht es doch, institutionelle Rahmungen gemeinsam mit den fragilen Bedingungen von Urteilen, Handeln und Öffentlichkeit zu erörtern. Ihre Ausführungen basieren auf der grundlegenden Unterscheidung zwischen drei Tätigkeiten und Modi des Weltbezugs. Während die Arbeit den Bezug zur objektiven Welt herstellt und dem biologischen Lebenszusammenhang dient, das Herstellen das zweckbestimmte Erzeugungen von Artefakten der materiellen und kulturell-symbolischen Welt bedeutet, ist einzig das Handeln eine gemeinsame weltgestaltende Tätigkeit. Das Handeln wird von Arendt als nicht determinierte Interaktion charakterisiert, die den gewohnten Verlauf der Welt unterbricht, indem das Handeln ihr etwas Neues hinzufügt (Arendt 2007, S. 166). In diesem Sinne versteht Arendt das Handeln als einzig genuin politische Tätigkeitsform, die keiner Notwendigkeit, auf die mit instrumentellen oder strategischen, effizienz- oder effektivitätsorientierten Überlegungen reagiert werden muss, unterliegt. Als Sinn des Handelns weist sie die Freiheit aus. Diese realisiert sich in einer inter-

\footnotetext{
${ }^{14}$ Die Perspektive Foucaults einzunehmen, ermöglicht, neoliberale Ideen und Motive von außen zu betrachten und darauf hin zu befragen, welche Figur der Freiheit sie tatsächlich, d.h. entgegen der neoliberalen Formulierungen realisieren.
} 
subjektiv vollzogenen Gestaltung der gemeinsam geteilten Welt, in der Menschen die Erfahrungen des „Nicht-gezwungen-Werden[s]“ machen (Arendt 1994, S. 201; zudem Arendt 2007, Kap. 5). Da die mit dem Begriff der politischen Freiheit fokussierte „kommunikativ erzeugte Macht“ (Habermas 1987, S. 231), die gemeinsam geteilte Welt zu gestalten, kein Vermögen Einzelner ist, beurteilt Arendt die Anderen sowie das Medium der Sprache grundlegend positiv und als konstitutive Momente des politischen Raumes. Dieser bildet sich, so Arendt, nur in einer plural verfassten Öffentlichkeit spontan und ungezwungen durch den Austausch und Streit divergierender Meinungen und Interessen der Handelnden heraus (Arendt 1994, S. 206; Brunkhorst 2011, S. 294). Das bedeutet zugleich, dass Handeln und Sprechen dabei wechselseitig aufeinander bezogen sind, eine intersubjektiv anerkannte Vorstellung des Guten zu formulieren (Arendt 2003, S. 7 f., 2007, S. $194 \mathrm{ff}$. sowie insgesamt Abschn. 2.7 und 5.24 und 5.25).

Eine unerlässliche Ressource der Verständigung ist Arendt zufolge die Urteilskraft, ein von Kant rekonstruiertes Erkenntnisvermögen, das sie politisch wendet. Insbesondere die reflektierende Dimension der Urteilskraft, die das Besondere nicht unter das Allgemeine subsumiert, sondern es als Besonderes (an-)erkennt, ist ihr zufolge wesentlich für Urteile, in denen Individuen hinsichtlich konkreter Themen und unter Absehung schematischer Anwendungen von Begriffen und Konzepten zu Entscheidungen gelangen (Arendt 2012). Sprechen, Handeln und Urteilen bilden einen Zusammenhang, der Arendt zufolge darauf angewiesen ist, zwischen Meinungen, Tatsachenwahrheiten und Interessen unterscheiden zu können (Arendt 1994, S. 327, 370). Die Perspektive anderer einzunehmen und als konstitutiv für die eigene Wahrnehmung zu erachten, sich also in Strukturen der Intersubjektivität zu bewegen, ist eine zentrale Voraussetzung dieser Unterscheidung (Meints-Stender 2019, S. 91ff.). In Ergänzung zu Arendt lässt sich hinzufügen, das freie Medien dafür essentiell sind, insofern sie die Möglichkeit einer transparenten Debatte bereitstellen (Anderson 2006).

Wird somit berücksichtigt, dass der Raum des Politischen absichernder Institutionen bedarf, soll die politische Freiheit nicht versiegen (Arendt 1968, Kap. 4), wird bereits nach dieser knappen Rekonstruktion der Überlegungen Arendts deutlich, dass sowohl die neoliberale Gesellschaft als auch diejenigen Gesellschaften, in denen sich Neoliberalismus und Datafizierung in einer teils widersprüchlichen Art verstricken, erhebliche Defizite aufweisen. Schon Arendts Krisendiagnose läuft darauf hinaus, dass der private den politischen Raum in der Moderne sukzessive verdrängt (Arendt 2007, Kap. 2). Der Neoliberalismus und dessen Transformation der Gesellschaft verschärfen diese Diagnose in einem Maße, das Arendt noch nicht vor Augen hatte. Tritt nun die Datafizierung hinzu, spitzt sich die Kritik weiter zu, selbst wenn in der - im Folgenden berücksichtigten - republikanischen Theoriediskussion durchaus positive Aspekte der Digitalisierung betont werden. Auf der ideellen Ebene liegt ein kategorialer Graben zwischen Urteilen sowie digitaler Mentalität, die die Welt einzig objektivistisch-szientistisch abzubilden vermag. In dieser Einstellung werden Handlungszusammenhänge mit Naturhaftigkeit identifiziert und Individuen objektiviert, denn einzig ihr regelmäßig beobachtbares Verhalten, das gemessen, analysiert, formalisiert und operationalisiert werden kann, wird wahrgenommen. Auf diesem Wege werden politisch handelnde Bürger*innen lediglich als heteronom bestimmte 
Spielbälle verstanden und dementsprechend manipuliert, demokratische Grundverständnisse hingegen infrage gestellt und Prozesse der Aushandlung technologischen Prozessen überlassen - eine fundamentale Entpolitisierung.

In der politikwissenschaftlichen, im weiteren Sinne philosophischen, gesellschafts- und kulturwissenschaftlichen Debatte werden jedoch einige (positiv gewertete) Potenziale digitaler Technologien diskutiert. So wird die Digitalisierung verschiedener Kommunikationsformen als push-Faktor begriffen, insofern sie die Interaktionskosten senkt, die Handlungsmotivation dadurch aber steigert und derart konnektives Handeln fördert (Thiel 2017, S. 204 f.) - ein Argument, das Grundannahmen der Rational Choice-Theorie übernimmt und auf politisches Handeln projiziert, ohne einen genuin politischen Begriff des Handelns zu explizieren. Dementsprechend verweist Antić (2017) auf die Zunahme an Möglichkeiten der Kommunikation über unterschiedliche Kanäle, die nicht in einer rationalistisch verengten Perspektive delegitimiert werden dürfen. Auch in diesem Sinne werden digitale Tools als Mittel des zivilen Ungehorsams gedeutet, der erst rückblickend normativ beurteilt werden könne (Züger et al. 2017; zum zivilen Ungehorsam Celikates 2010). Gerbaudo (2017) begreift digitale Kommunikationsmittel als präfigurative Protestelemente, die der Bildung neuer Formen kommunikativer Selbstbestimmung dienen können. Stalder (2017) behauptet gar, dass eine digitale Referenzialität als kulturelle Ressource zu verstehen ist, die dem Aufbau neuer Formen von Gemeinschaftlichkeit dient, was Thiel (2017, S. 207 f.) als republikanisches Ideal deutet.

Diese Urteile gründen in der Fokussierung der Nutzung digitaler Technologien, die jedoch deren agency unzureichend berücksichtigt. Der Datafizierungsbegriff lenkt hingegen den Blick auf die soziotechnische Ordnung, deren grundlegender Mechanismus den Phänomenbereich auf unterschiedlichen Ebenen prägt, d.h. die trotz besonderer Nutzungsweisen und -interessen allgemeine Tendenzen auf der Ebene der Strukturen, in denen interagiert wird, auf der Ebene der Inhalte, die Interaktionen motivieren, und auf der Ebene der Subjektivierung hervorrufen (Passoth und Rammert 2019, S. 149f. und Fn. 7; benannte Ebene nun in der Reihenfolge). Paradigmatisch zeigt sich diese Wirkung datafizierter Ordnungen, innerhalb derer Technologien und soziale Interaktionen spezifisch verkoppelt werden, in den Sozialen Medien. Diese weisen enorm ausgeprägte kapitalistische und heteronome Strukturen auf, da die Plattformen und die produzierten Daten Eigentum marktdominierender Unternehmen sind, die im Hintergrund die eigentlichen Profiteure der Interaktionen sind. Interaktionen wiederum treiben die Akkumulation von Daten voran und lassen sich schwerlich als normativ gehaltvolle Partizipationsformen verstehen (Buchstein 1997; Galloway und Thacker 2010; Maschewski und Nosthoff 2018, S. 17). Ein öffentlicher Raum jedoch, der zum privaten Besitz wird, wird entpolitisiert (Arendt 2007, S. 60-66). Hinzukommt, dass die Möglichkeit der Überwachung den Interaktionsraum mindert, weil sich gerade User*innen aus dem Feld sozialer Proteste der Gefahr bewusst sind, dass ihre Profile über einen langen Zeitraum von Sicherheitsorganen abrufbar sind, oder aber weil über die Auswertung 
von Suchabfragen sozialer Protest frühzeitig vorhergesagt und dagegen vorgegangen werden kann (Stahl 2016). ${ }^{15}$

Zudem werden die Kommunikationsformen in Sozialen Medien durch das Interface und die Kommunikationsmöglichkeiten durch Algorithmen determiniert, weisen also einen Zwangscharakter auf, der gerade als das Gegenteil von Spontanität zu begreifen ist (Maschewski und Nosthoff 2018, S. 12). Diese „Tendenzen der Automatisierung politischen Handelns“ (Züger et al. 2017, S. 275) stehen der Erfahrung politischer Freiheit diametral entgegen. Ebenso ist fraglich, inwiefern der Raum, den Soziale Medien, generell digitale Technologien erschließen, durch das Faktum der Pluralität charakterisiert werden kann. Unter den Begriffen „Filter Bubble“ und „Echokammer“ haben jüngst algorithmisch produzierte Effekte eine steile Karriere gemacht, die hier auf einen problematischen Aspekt hinzuweisen vermögen, obzwar sie zunächst lediglich eine theoretische Hypothese darstellen. Filterblasen sind voneinander isolierte Bereiche digitaler Öffentlichkeit, zwischen denen kein Austausch (bspw. über divergierende Meinungen und Interessen) besteht. Zu Echokammern werden sie, sobald der Mangel an Austausch dazu führt, dass sich bestehende Meinungen und Präferenzen nicht allein verfestigen, sondern sich gar wechselseitig verstärken (Pariser 2012); ein Phänomen, das gegenwärtig mit Blick auf sogenannte Corona-Demos und Radikalisierungstendenzen in Chatgruppen diskutiert wird (Linder 2020). Sicherlich hat die empirische Medienforschung noch keine abschließenden Resultate über das tatsächliche Ausmaß von Filterblasen und Echokammern erbracht, u. a. weil entsprechende Daten (bisher) nur den privaten Anbieter*innen zur Verfügung stehen und nicht umfassend ausgewertet werden konnten (Stark 2019). Zumindest ,[e]inige Studien deuten auf einen eher moderaten Effekt von algorithmischer Kuratierung bei Plattformen wie Google und Facebook hin“ (Rau und Stier 2019, S. 413). Skeptischer stimmt der Hinweis, dass Soziale Medien lediglich schwache Bindungen zu etablieren vermögen, innerhalb derer eine geringe Anzahl an User*innen kommuniziert, während ein Großteil lediglich konsumiert, sodass jene Medien einem arendtianischen Verständnis politischen Handelns nicht genügen (Schwarz 2014, S. 182-185). Zumindest mit Blick auf das Feld der Politik muss daher das vorsichtig formulierte Urteil erlaubt sein, dass die technologisch angelegten Tendenzen einer pluralen diskursiven Verständigung über die gemeinsamen Belange nicht gerade förderlich sind - selbst dann, wenn andere Variablen hier ebenfalls einen Einfluss auszuüben vermögen (Rau und Stier 2019, S. 413). ${ }^{16}$

Hier ist nun der Schritt von einer Problematisierung der technologisch-kapitalistischen Struktur zur Reflexion der Inhalte, die in datafizierten Kontexten produziert und getauscht werden, vollzogen. Im US-amerikanischen Wahlkampf zur Wahl 2016 wurden datenbasierte Nutzer*innenanalysen eingesetzt, um Desinformationen gezielt zu adressieren und derart Wahlentscheidungen zu manipulieren oder gar ganze

\footnotetext{
15 Zum digitalen Überwachungskapitalismus Zuboff (2018); zur Möglichkeit, (in diesem Falle nicht politischen Protest, sondern) Grippewellen durch die Analyse von Suchabfragen vorherzusagen Ginsberg et al. (2009).

16 Auch wenn angemerkt werden kann, dass Öffentlichkeit immer schon fragmentiert war (Negt und Kluge 1976), ändert das nichts daran, dass digitale Technologien aufgrund von Daten und Algorithmen Formen diskursiver Argumentation über verschiedene Meinungen eher unterminieren als sie zu fördern.
} 
Wähler*innengruppen von Wahlen auszuschließen (Citron und Chesney 2018; Jamieson 2018; Stengel 2019). Gewiss, strategisch-mediale Manipulationsversuche sind kein Novum, doch ermöglicht die digitale Datafizierung ihren passgenauen Einsatz. Da sie die Bedingungen der Möglichkeit der Wahrheitsproduktion unterminieren, lassen sich die für Soziale Medien typischen fake news als Moment verstehen, das grundlegend den Zusammenhang von Urteilen, Sprechen und Handeln zerreißt (Lüdemann 2020). Es ist somit nicht allein fraglich, ob digitale Öffentlichkeiten als Raum politischer Freiheit begriffen werden können, sondern inwiefern fake news über den digitalen Bereich hinaus den gesamten Raum des Politischen erodieren lassen können (systematisch zur Lüge im Politischen: König 2020).

Auch für den Prozess politischer Subjektivierung lassen sich abschließend Tendenzen politiktheoretisch reflektieren, die in datafizierten Strukturen gründen. In der republikanisch orientierten Theorietradition wird argumentiert, dass die Einbindung in öffentliche Diskussions- und Kooperationsstrukturen Individuen politisch subjektiviert, weil sie gerade dort die Erfahrung politischer Freiheit machen (Sunstein 2009). Diese Erfahrungen politischen Handelns sind als unmittelbar eigene Erfahrungen oder als narrative tradierte Momente einer gemeinsam geteilten Geschichte $\mathrm{zu}$ verstehen, die wiederum ein konstitutives Moment politischer Subjektivität darstellt (Straßenberger 2005, S. 27-31). Die serielle datenbasierte Zusammenstellung von Inhalten in Sozialen Medien sowie die verschärfte Konkurrenz innerhalb der digitalen Aufmerksamkeitsökonomie genügen jedoch nicht der von Arendt fokussierten erzählerischen Qualität. Eine retrospektive Narration steht geradezu in fundamentalem Widerspruch zur zwar um Abgrenzung bemühten, jedoch oftmals standardisierten (Selbst-)Darstellung sowie der automatisierten und von Algorithmen sortierten Präsentation von Inhalten. ${ }^{17}$ Gewichtiger wird das Argument, wird zudem der Gedanke hinzugezogen, dass sich die bereits mit Blick auf die postmoderne Kultur thematisierte zerrissene Zeiterfahrung und das Erleben der Welt in kurzen zusammenhangslosen Zeitintervallen (Jameson 1986, S. 71) in datafizierten Kontexten verschärfen, insofern dort schwerer jene dauerhaften Bindungen zu anderen aufgebaut werden können, die als Grundlage einer gemeinsam erlebten Welt zu verstehen sind (Rosa 2020). Es fehlt datafizierten Strukturen daher an Momenten, Erfahrungen politischer Freiheit narrativ einzuholen und so Prozesse politischer Subjektivierung anzustoßen. Unter der Annahme, dass die Erfahrungen politischer Freiheit ein konstitutives Moment der Motivation politischen Handelns sind (Straßenberger 2005, S. 41-51 nach Arendt), können jene Strukturen die Voraussetzungen politischen Handelns nicht bereitstellen. „[D]ass niemand frei ist, der nicht aus der Erfahrung weiß, was öffentliche Freiheit ist" (Arendt 1968, S. 326), bedeutet, dass datafizierte Kontexte einer republikanisch verstandenen Freiheit nicht nur nicht förderlich sind, sondern ihr Fundament tendenziell zu untergraben drohen.

17 So die Social Media-Analysen von Martínez und Weixler (2019). Vgl. Reckwitz (2017), Kap. IV zur digitalen Aufmerksamkeitsökonomie. 


\section{Ausblick: Ein Schritt zum Handeln}

Im vorliegenden Text wurden die politische Philosophie des Neoliberalismus sowie die digitale Mentalität dargestellt. Sowohl Gemeinsamkeiten - in Hinblick auf den Wissensbegriff - als auch Widersprüche - zwischen ungeplanter Ordnungsbildung auf der einen, algorithmischen Prozessen und datenbasierten Steuerungskonzepten auf der anderen Seite - wurden dabei rekonstruiert. Ihre praktische Entfaltung in sozialen Zusammenhängen wurde in republikanischer Perspektive diskutiert. Abschließend möchte ich einen skizzenhaften Ausblick geben, was politisches Handeln in Anbetracht einer datafizierten neoliberal Gesellschaft bedeuten kann.

Meine Überlegungen führen zunächst dazu, dass ein digitales politisches Handeln letztendlich ein aporetischer Begriff ist, weil er Begriffsmomente vereint, die sich wechselseitig ausschließen. Das bedeutet zugleich, dass immanent agierende Praktiken, die durch den Einsatz digitaler Technologien datafizierende Kontexte zu transformieren versuchen, als paradoxal gelten müssen, da sie diese Kontexte aufgrund der Erzeugung weiterer automatisch verarbeiteter Daten reproduzieren. Eine „Steuerungswende“ (so der Titel von Schaupp und Jochum 2019) hin zu einer demokratisch-nachhaltigeren Wirtschaftsplanung oder Bestrebungen, Daten einzig durch innovative Nutzungsordnungen allen zu Zwecken gesteigerter Wertschöpfungsprozesse zugänglich zu machen (Mayer-Schönberger und Ramge 2020), erweisen sich vor dem Hintergrund des vorliegenden Textes als Ideen, die nicht im Stande sind, auf das genuin politische Defizit des beschriebenen Prozesses zu reagieren. Hier nehmen sich die bereits thematisierten politikwissenschaftlichen Perspektiven nicht aus, die sich primär auf die Nutzung der Technologien beziehen - aber eher randständig thematisieren, was überhaupt digitalisiert bzw. datafiziert wird.

Daher, so meine abschließenden Überlegungen, sind zum einen die sozialen Konflikte zu fokussieren, innerhalb derer soziale und politische Zusammenhänge gegen die Übergriffe der (mit neoliberalen Facetten versehenen) Datafizierung verteidigt werden. Über eine derart defensive Position hinausgehend, die lediglich Residuen bewahren will, stellt sich in einer republikanischen Perspektive jedoch zum anderen die Frage, inwiefern der Digitalisierungsprozess insgesamt zu politisieren ist, sollen digitale Technologien nicht per se als zu bekämpfendes Problem ausgemacht und lediglich eine Aporie im Begriff digitalen politischen Handelns diagnostiziert werden. Wird die partizipative Gestaltung jenes Prozesses zum Ziel erklärt, werden Algorithmen transparent und kooperativ gestaltet und zugleich solutionistisches und behavioristisches Denken zurückgewiesen, rückt eine dezidiert politische, d.h. kollektive Autonomie fördernde Kopplung der technologischen, sozialen und politischen Sphäre in den Bereich des Möglichen. Innovative Wege sind dabei zu gehen, experimentelle Formen (wie bspw. Zukunftswerkstätten oder Hackathons) zu erproben, um negative und positive Freiheitsrechte in einem institutionellen Design der Technologiengenese (Häußling 2014) zu verschränken. Ein derart von der politischen Gemeinschaft angeeigneter Digitalisierungsprozess bietet durchaus Potenziale der wechselseitigen Vernetzung und kommunikativen Handlungskoordination, die dann ggf. gar in der Lage sind, den neoliberalen Kontext der Nutzung digitaler Technologien durch politisches Handeln zu transformieren. Die politische Theorie kann hier als Bündnispartnerin agieren, indem a) auf epistemischer Ebene die digitale Menta- 
lität als Entpolitisierung, b) auf funktionaler Ebene die Datafizierung als zusätzlicher Pfeiler einer Kapital akkumulierenden Gesellschaft sowie c) die widersprüchliche Verbindung von Neoliberalismus und digitaler Mentalität als Herausbildung eines Herrschaftsprojekts thematisiert werden (zu dieser Unterscheidung Celikates 2017, S. 62f.). Der vorliegende Text versteht sich als ein Schritt in diese Richtung.

Funding Open Access funding enabled and organized by Projekt DEAL.

Open Access Dieser Artikel wird unter der Creative Commons Namensnennung 4.0 International Lizenz veröffentlicht, welche die Nutzung, Vervielfältigung, Bearbeitung, Verbreitung und Wiedergabe in jeglichem Medium und Format erlaubt, sofern Sie den/die ursprünglichen Autor(en) und die Quelle ordnungsgemäß nennen, einen Link zur Creative Commons Lizenz beifügen und angeben, ob Änderungen vorgenommen wurden.

Die in diesem Artikel enthaltenen Bilder und sonstiges Drittmaterial unterliegen ebenfalls der genannten Creative Commons Lizenz, sofern sich aus der Abbildungslegende nichts anderes ergibt. Sofern das betreffende Material nicht unter der genannten Creative Commons Lizenz steht und die betreffende Handlung nicht nach gesetzlichen Vorschriften erlaubt ist, ist für die oben aufgeführten Weiterverwendungen des Materials die Einwilligung des jeweiligen Rechteinhabers einzuholen.

Weitere Details zur Lizenz entnehmen Sie bitte der Lizenzinformation auf http://creativecommons.org/ licenses/by/4.0/deed.de.

\section{Literatur}

Aamodt, Agnar, und Mads Nygård. 1995. Different roles and mutual dependencies of data, information, and knowledge - an AI perspective on their integration. Data and Knowledge Engineering 16:191-222.

Anderson, Elizabeth. 2006. The epistemology of democracy. Episteme 3:8-22.

Antić, Andreas. 2017. Digitale Öffentlichkeiten im demokratischen Experimentalismus. In Politische Theorie und Digitalisierung, Hrsg. Daniel Jacob, Thorsten Thiel, 139-160. Baden-Baden: Nomos.

Arendt, Hannah. 1968. Über die Revolution. München: Piper.

Arendt, Hannah. 1994. Zwischen Vergangenheit und Zukunft. Übungen im politischen Denken. München: Piper.

Arendt, Hannah. 2003. Denktagebuch. 1950 bis 1973. München: Piper.

Arendt, Hannah. 2007. Vita activa oder Vom tätigen Leben. München: Piper.

Arendt, Hannah. 2012. Das Urteilen. München: Piper.

Bächle, Thomas Christian. 2016. Digitales Wissen, Daten und Überwachung zur Einführung. Hamburg: Junius.

Baecker, Dirk. 2018. 4.0 oder Die Lücke die der Rechner lässt. Leipzig: Merve.

Barbrook, Richard, und Andy Cameron. 1996. The Californian ideology. Science as Culture 6:44-72.

Barlösius, Eva. 2001. Die Macht der Repräsentation. In Gesellschaftsbilder im Umbruch. Soziologische Perspektiven in Deutschland, Hrsg. Eva Barlösius, et al., 179-202. Wiesbaden: VS.

Becker, Gary Stanley. 1993. Der ökonomische Ansatz zur Erklärung menschlichen Verhaltens. Tübingen: Mohr Siebeck.

Benjamin, Walter. 1991. Abhandlungen. Gesammelte Schriften, Bd. I. Frankfurt a.M.: Suhrkamp.

Berg, Sebastian, und Thorsten Thiel. 2019. Widerstand und die Formierung von Ordnung in der digitalen Konstellation. Zeitschrift für Politische Theorie 10:67-86.

Berg, Sebastian, Niklas Rakowski, und Thorsten Thiel. 2020. Die digitale Konstellation. Eine Positionsbestimmung. Zeitschrift für Politikwissenschaft 30:171-191.

Bevir, Mark, und Kim McKee. 2016. Governance jenseits des Neoliberalismus in Großbritannien. In Der Staat des Neoliberalismus, Hrsg. Thomas Biebricher, 209-236. Baden-Baden: Nomos.

Biebricher, Thomas. 2016. Einleitung: Neoliberalismus und Staat - ziemlich beste Feinde. In Der Staat des Neoliberalismus, Hrsg. Thomas Biebricher, 9-28. Baden-Baden: Nomos.

Biebricher, Thomas. 2021. Die politische Theorie des Neoliberalismus. Berlin: Suhrkamp.

von Bismarck, Julius. 2019. Public face. ARCH 52:144-147. 
Boas, Taylor C., und Jordan Gans-Morse. 2009. Neoliberalism. From new liberal philosophy to anti-liberal slogan. Studies in Comparative 44:137-161.

Bock, Wolfgang. 2019. Neue Medien und Ideologie. Zur Dialektik der digitalisierten Aufklärung. In Handbuch Kritische Theorie, Hrsg. Uwe H. Bittlingmayer, et al., 1213-1246. Wiesbaden: Springer VS.

Boltanski, Luc, und Ève Chiapello. 2003. Der neue Geist des Kapitalismus. Konstanz: UVK.

Borgwardt, Angela. 2018. Digitalisierung in der Wissenschaft. Berlin: Friedrich-Ebert-Stiftung.

Boyd, Danah, und Kate Crawford. 2013. Big Data als kulturelles, technologisches und wissenschaftliches Phänomen. In Big Data. Das neue Versprechen der Allwissenheit, Hrsg. Heinrich Geiselberger, Tobias Moorstedt, 187. Berlin: Suhrkamp.

Bröckling, Ulrich. 2007. Das unternehmerische Selbst. Soziologie einer Subjektivierungsform. Frankfurt a.M.: Suhrkamp.

Brown, Wendy. 2015. Die schleichende Revolution. Wie der Neoliberalismus die Demokratie zerstört. Berlin: Suhrkamp.

Brown, Wendy. 2019. Das Monster des Neoliberalismus. Autoritäre Freiheit in den „Demokratien“ des 21. Jahrhunderts. In Kritische Theorie der Politik, Hrsg. Ulf Bohmann, Paul Sörensen, 539-576. Berlin: Suhrkamp.

Brunkhorst, Hauke. 2011. Macht/Gewalt/Herrschaft. In Arendt-Handbuch. Leben, Werk, Wirkung, Hrsg. Wolfgang Heuer, et al., 294-298. Stuttgart: Metzler.

Buchstein, Hubertus. 1997. Bytes that bite. The Internet and deliberative democracy. Constellations 4:248-263.

Buckermann, Paul, Simon Schaupp, und Anne Koppenburger (Hrsg.). 2017. Kybernetik, Kapitalismus, Revolutionen. Emanzipatorische Perspektiven im digital-technologischen Wandel. Münster: UNRAST.

Butterwegge, Christoph, Bettina Lösch, und Ralf Ptak. 2016. Kritik des Neoliberalismus. Wiesbaden: VS.

Candeias, Mario. 2009. Neoliberalismus - Hochtechnologie - Hegemonie. Grundrisse einer transnationalen kapitalistischen Produktions- und Lebensweise. Eine Kritik. Hamburg: Argument.

Castells, Manuel. 2000. The rise of the network society. Oxford: Blackwell Publishers.

Celikates, Robin. 2010. Ziviler Ungehorsam und Radikale Demokratie. In Das Politische und die Politik, Hrsg. Thomas Bedorf, Kurt Röttgers, 274-300. Berlin: Suhrkamp.

Celikates, Robin. 2017. Epistemische Ungerechtigkeit, Loopingeffekte und Ideologiekritik. Eine sozialphilosophische Perspektive. WestEnd. Neue Zeitschrift für Sozialforschung 14:54-72.

Celikates, Robin. 2019. Moralischer Fortschritt, soziale Kämpfe und Emanzipationsblockaden: Elemente einer Kritischen Theorie der Politik. In Kritische Theorie der Politik, Hrsg. Ulf Bohmann, Paul Sörensen, 397-425. Berlin: Suhrkamp.

Citron, Danielle K., und Robert Chesney. 2018. Deep fakes: a looming challenge for privacy, democracy, and national security national security. Draft (2018). Boston: Boston University School of Law Boston.

Crouch, Colin. 2008. Postdemokratie. Bonn: Bundeszentrale für politische Bildung.

Crouch, Colin. 2011. Das befremdliche Überleben des Neoliberalismus. Postdemokratie II. Berlin: Suhrkamp.

Dörre, Klaus. 2009. Die neue Landnahme. Dynamiken und Grenzen des Finanzmarktkapitalismus. In Soziologie - Kapitalismus - Kritik. Eine Debatte, Hrsg. Klaus Dörre, et al., 21-86. Frankfurt a.M.: Suhrkamp.

Foucault, Michel. 2005. Schriften in vier Bänden. Frankfurt a.M.: Suhrkamp.

Foucault, Michel. 2006. Die Geburt der Biopolitik. Geschichte der Gouvernementalität II. Frankfurt a.M.: Suhrkamp.

Friedman, Milton. 2005. Kapitalismus und Freiheit. München: Piper.

Friedman, Milton. 1970. Was die Aufgabe von Unternehmen ist - und was nicht (Teil 2). Ludwig von Mises Institut Deutschland. https://www.misesde.org/2016/01/was-die-aufgabe-von-unternehmen-ist-\%e2 \%80\%93-und-was-nicht-teil-2/. Zugegriffen: 8. Okt. 2020.

Galloway, Alexander R., und Eugene Thacker. 2010. The exploit. A theory of networks. Minneapolis: University of Minnesota Press.

Gerbaudo, Paolo. 2017. From cyber-autonomism to cyber-populism. An ideological analysis of the evolution of digital activism. triple C: Communication, Capitalism \& Critique 15:477-489.

Ginsberg, J., M.H. Mohebbi, R.S. Patel, L. Brammer, M.S. Smolinski, und L. Brilliant. 2009. Detecting influenza epidemics using search engine query data. Nature 457:1012-1015.

Habermas, Jürgen. 1981a. Handlungsrationalität und gesellschaftliche Rationalisierung. Theorie des kommunikativen Handelns, Bd. 1. Frankfurt am Main: Suhrkamp.

Habermas, Jürgen. 1981b. Zur Kritik der funktionalistischen Vernunft. Theorie des kommunikativen Handelns, Bd. 2. Frankfurt a.M.: Suhrkamp. 
Habermas, Jürgen. 1987. Philosophisch-politisch Profile. Frankfurt a.M.: Suhrkamp.

Habermas, Jürgen. 2009. Sprachtheoretische Grundlegung der Soziologie. Philosophische Texte. Bd. 1. Frankfurt a.M.: Suhrkamp.

Hartmann, Martin. 2018. Vorsicht, Vorsicht und nochmals Vorsicht? Zur Auseinandersetzung um den Begriff und das Phänomen des Neoliberalismus. WestEnd. Neue Zeitschrift für Sozialforschung 15:143-152.

Harvey, David. 2011. A brief history of neoliberalism. Oxford: Oxford University Press.

Häußermann, Hartmut, Dieter Läpple, und Walter Siebel. 2008. Stadtpolitik. Frankfurt a.M.: Suhrkamp.

Häußling, Roger. 2014. Design als soziotechnische Relation. Neue Herausforderungen der Gestaltung inter- und transaktiver Technik am Beispiel humanoider Robotik. In Das Design der Gesellschaft, Hrsg. Stephan Moebius, Sophia Prinz, 263-288. Bielefeld: transcript.

Häußling, Roger. 2020. Daten als Schnittstellen zwischen algorithmischen und sozialen Prozessen. Konzeptuelle Überlegungen zu einer Relationalen Techniksoziologie der Datafizierung in der digitalen Sphäre. In Soziologie des Digitalen - Digitale Soziologie? Soziale Welt - Sonderband 23., Hrsg. Sabine Maasen, Jan-Hendrik Passoth, 134-150. Baden Baden: Nomos.

Häußling, Roger, Michael Eggert, Daniel Kerpen, Jacqueline Lemm, Niklas Strüver, und Nenja Katharina Ziesen. 2017. Schlaglichter der Digitalisierung. Virtureale(r) Körper - Arbeit - Alltag: Ein Vorsto $\beta$ zum Kern der Digitalisierung aus einer techniksoziologisch-relationalen Perspektive. Aachen: RWTH Publications.

von Hayek, Friedrich A. 1948. Wahrer und falscher Individualismus. ORDO - Jahrbuch für die Ordnung von Wirtschaft und Gesellschaft 1:19-55.

von Hayek, Friedrich A. 1963. Arten der Ordnung. ORDO - Jahrbuch für die Ordnung von Wirtschaft und Gesellschaft 14:3-20.

von Hayek, Friedrich A. 1975. Die Anmaßung von Wissen. ORDO - Jahrbuch für die Ordnung von Wirtschaft und Gesellschaft 26:12-21.

von Hayek, Friedrich A. 2003a. Der Weg zur Knechtschaft. München: Olzog.

von Hayek, Friedrich A. 2003b. Recht, Gesetz und Freiheit. Eine Neufassung der liberalen Grundsätze der Gerechtigkeit und der politischen Ökonomie. Tübingen: Mohr Siebeck.

Hayek Friedrich von, August. 1991. Die Verfassung der Freiheit. Tübingen: Mohr.

Hayek Friedrich von, August. 1994. Freiburger Studien. Gesammelte Aufsätze. Tübingen: Mohr Siebeck.

Helbing, Dirk. 2015. Thinking ahead. Essays on big data, digital revolution, and participatory market society. Wiesbaden: Springer VS.

Honneth, Axel. 2016. Neoliberalismus? Eine skeptische Wortmeldung anlässlich einer Studie von David M. Kotz. WestEnd. Neue Zeitschrift für Sozialforschung 13:167-179.

Houben, Daniel. 2018. Instrumentelle Vernunft in der Datengesellschaft - Zur Relevanz der Kritischen Theorie für das Verständnis der Datafizierung des Sozialen. In Digitale Transformation im Diskurs. Kritische Perspektiven auf Entwicklungen und Tendenzen im Zeitalter des Digitalen, Hrsg. Christian Leineweber, Claudia de Witt, 197-220. Hagen: FernUniversität.

Hoye, J. Matthew, und Jeffrey Monaghan. 2018. Surveillance, freedom and the republic. European Journal of Political Theory 17:343-363.

Hutchby, Ian. 2016. Technologies, texts and affordances. Sociology 35:441-456.

Jacob, Daniel, und Thorsten Thiel (Hrsg.). 2017. Politische Theorie und Digitalisierung. Baden-Baden: Nomos.

Jameson, Fredric. 1986. Postmoderne - zur Logik der Kultur im Spätkapitalismus. In Postmoderne. Zeichen eines kulturellen Wandels, Hrsg. Andreas Huyssen, Klaus R. Scherpe, 45-102. Reinbek bei Hamburg: Rowohlt.

Jamieson, Kathleen Hall. 2018. Cyberwar. How Russian hackers and trolls helped elect a president: what we don't, can't, and do know. New York: Oxford University Press.

Kitchin, Rob. 2014. The data revolution. Big data, open data, data infrastructures \& their consequences. London: SAGE.

Knuth, Donad E., und Luis Trabb Pardo. 1978. The early development of programming languages. In A history of computing in the twentieth century. A collection of essays with introductory essay and indexes, Hrsg. Nicholas C. Metropolis, 197-273. Orlando: Academic Press.

König, Helmut. 2020. Lüge und Täuschung in den Zeiten von Putin, Trump \& Co. Bielefeld: transcript.

Kurz, Constanze. 2017. „Pre-Crime“: Über Menschen, die ungewollt Teil von Datenexperimenten sind. Netzpolitik. https://netzpolitik.org/2017/pre-crime-ueber-menschen-die-ungewollt-teil-vondatenexperimenten-sind/. Zugegriffen: 28. Dez. 2019.

Latour, Bruno. 1996. On actor-network theory. A few clarifications. Soziale Welt 47:369-382.

Lindemann, Gesa. 2020. Die Brutalität der Codes. Soziologische Revue 43:301-311. 
Lippmann, Walter. 1945. Die Gesellschaft freier Menschen. Bern: Francke.

Lippmann, Walter. 1973. An inquiry into the principles of the good society. Westport: Greenwood Press.

Lüdemann, Susanne. 2020. Demokratie und Urteilskraft im digitalen Zeitalter. Ein Selbstversuch. WestEnd. Neue Zeitschrift für Sozialforschung 17:3-24.

Mämecke, Thorben, Jan-Hendrik Passoth, und Josef Wehner. 2018. Bedeutende Daten - Einführende Überlegungen. In Bedeutende Daten. Modelle, Verfahren und Praxis der Vermessung und Verdatung im Netz, Hrsg. Thorben Mämecke, et al., 1-14. Wiesbaden: Springer VS.

Martínez, Matías, und Antonius Weixler. 2019. Selfies und Stories. Authentizität und Banalität des narrativen Selbst in Social Media. DIEGESIS. Interdisziplinäres E-Journal für Erzählforschung 8:49-67.

Maschewski, Felix, und Anna-Verena Nosthoff. 2018. „Passivität im Kostüm der Aktivität“. Über Günther Anders' Kritik kybernetischer Politik im Zeitalter der ,totalen Maschine“. BEHEMOTH—A Journal on Civilisation 11:8-25.

Mau, Steffen. 2017. Das metrische Wir. Über die Quantifizierung des Sozialen. Berlin: Suhrkamp.

Mau, Steffen. 2019. Lütten Klein. Leben in der ostdeutschen Transformationsgesellschaft. Berlin: Suhrkamp.

Mayer-Schönberger, Viktor, und Kenneth Cukier. 2013. Big data. A revolution that will transform how we live, work, and think. Boston: Houghton Mifflin Harcourt.

Mayer-Schönberger, Viktor, und Thomas Ramge. 2020. Machtmaschinen. Warum Datenmonopole unsere Zukunft gefährden und wie wir sie brechen. Hamburg: Murmann.

Mayntz, Renate. 2004. Governance im modernen Staat. In Governance - Regieren in komplexen Regelsystemen. Eine Einführung, Hrsg. Arthur Benz, 65-76. Wiesbaden: VS.

Meints-Stender, Waltraud. 2019. Gesellschaftliche und politische Macht. In Macht und Gewalt. Hannah Arendts ,On Violence“ neu gelesen, Hrsg. Mirko Wischke, Georg Zenkert, 77-96. Wiesbaden: Springer.

Mersch, Dieter. 2019. Ideen zu einer Kritik ,,algorithmischer“ Rationalität. Deutsche Zeitschrift für Philosophie 67:851-873.

Morozov, Evgeny. 2014. To save everything, click here. The folly solutionism. London: PublicAffairs.

Morozov, Evgeny. 2013. „We are abandoning all the checks and balances“. The Guardian online. https://www.theguardian.com/technology/2013/mar/09/evgeny-morozov-technology-solutionisminterview. Zugegriffen: 21. Apr. 2017.

Morozov, Evgeny. 2016. Vom Global Village zum Feudalstaat. Neue Zürcher Zeitung. https://www.nzz.ch/ feuilleton/zeitgeschehen/evgeny-morozov-ueber-das-internet-vom-global-village-zum-feudalstaat1d.113600. Zugegriffen: 2. Okt. 2020.

Münch, Richard. 2011. Akademischer Kapitalismus. Über die politische Ökonomie der Hochschulreform. Berlin: Suhrkamp.

Nachtwey, Oliver, und Timo Seidl. 2017. Die Ethik der Solution und der Geist des digitalen Kapitalismus. IfS Working Paper \#11. Frankfurt a.M.: Institut für Sozialforschung.

Nachtwey, Oliver, und Philipp Staab. 2015. Die Avantgarde des digitalen Kapitalismus. Mittelweg 36:1-21. Nassehi, Armin. 2019. Muster. Theorie der digitalen Gesellschaft. München: C. H. Beck.

Negt, Oskar, und Alexander Kluge. 1976. Öffentlichkeit und Erfahrung. Zur Organisationsanalyse von bürgerlicher und proletarischer Öffentlichkeit. Frankfurt a.M.: Suhrkamp.

Nosthoff, Anna-Verena, und Felix Maschewski. 2017. „Democracy as Data“? Über Cambridge Analytica und die „moralische Phantasie“. Merkur. Deutsch Zeitschrift für europäisches Denken. https://www.merkur-zeitschrift.de/2017/02/06/democracy-as-data-ueber-cambridge-analytica-unddie-moralische-phantasie/. Zugegriffen: 31. März 2017.

Nozick, Robert. 2006. Anarchie, Staat, Utopia. München: Olzog.

Pariser, Eli. 2012. Filter Bubble. Wie wir im Internet entmündigt werden. München: Hanser.

Passoth, Jan-Hendrik, und Werner Rammert. 2019. Fragmentale Differenzierung als Gesellschaftsdiagnose. Was steckt hinter der zunehmenden Orientierung an Innovation, Granularität und Heterogenität? In Berliner Schlüssel zur Techniksoziologie, Hrsg. Cornelius Schubert, Ingo Schulz-Schaeffer, 143-177. Wiesbaden: Springer.

Peck, Jamie. 2016. Pushing Austerity: Staatsversagen, bankrotte Kommunen und die Krisen des fiskalischen Föderalismus in den Vereinigten Staaten. In Der Staat des Neoliberalismus, Hrsg. Thomas Biebricher, 237-262. Baden-Baden: Nomos.

Pettit, Philip. 1997. Republicanism. A theory of freedom and government. Oxford: Oxford University Press.

Prietl, Bianca, und Daniel Houben. 2018. Einführung. Soziologische Perspektiven auf die Datafizierung der Gesellschaft. In Datengesellschaft, Hrsg. Bianca Prietl, 7-32. Bielefeld: transcript. 
Ptak, Ralf. 2016. Das Staatsverständnis im Ordoliberalismus. Eine theoriegeschichtliche Analyse mit aktuellem Ausblick. In Der Staat des Neoliberalismus, Hrsg. Thomas Biebricher, 31-73. Baden-Baden: Nomos.

Rau, Jan Philipp, und Sebastian Stier. 2019. Die Echokammer-Hypothese. Fragmentierung der Öffentlichkeit und politische Polarisierung durch digitale Medien? Zeitschrift für Vergleichende Politikwissenschaft 13:399-417.

Reckwitz, Andreas. 2017. Die Gesellschaft der Singularitäten. Zum Strukturwandel der Moderne. Berlin: Suhrkamp.

Reichert, Ramón. 2014. Einführung. In Big Data, Hrsg. Ramón Reichert. Bielefeld: transcript.

Richter, Emanuel. 2018. Republikanismus. In Handbuch Politische Ideengeschichte. Zugänge - Methoden - Strömungen, Hrsg. Samuel Salzborn, 208-219. Stuttgart: J.B. Metzler.

Rosa, Hartmut. 2020. Von summenden und verstummenden Resonanzachsen im Zeitalter der Digitalisierung. In Resonanz und Lebensqualität. Weltbeziehungen in Zeiten der Digitalisierung. Pädagogische Perspektiven, Hrsg. Edwin Hübner, Leonhard Weiss, 9-12. Leverkusen: Budrich.

Rutishauser, Heinz. 1956. Maßnahmen zur Vereinfachung von Rechenplänen bei elektronischen Rechenanlagen. In Elektronische Rechenmaschinen und Informationsverarbeitung, Hrsg. Alwin Walther, Walter Hoffmann, 26-30. Braunschweig: Vieweg.

Schaupp, Simon, und Georg Jochum. 2019. Die Steuerungswende. Zur Möglichkeit einer nachhaltigen und demokratischen Wirtschaftsplanung im digitalen Zeitalter. In Marx und die Roboter. Vernetzte Produktion, Künstliche Intelligenz, und lebendige Arbeit, Hrsg. Sabine Nuss, Florian Butollo, 327-344. Berlin: Dietz.

Schaupp, Simon. 2017. Vergessene Horizonte. Der kybernetische Kapitalismus und seine Alternativen. In Kybernetik, Kapitalismus, Revolutionen. Emanzipatorische Perspektiven im digital-technologischen Wandel, Hrsg. Paul Buckermann, et al., 51-73. Münster: UNRAST.

Schmidt, Hermann. 1941. Denkschrift zur Gründung eines Instituts für Regelungstechnik. Berlin: VDI.

Schnell, Rainer, Paul Bernhard Hill, und Elke Esser. 2005. Methoden der empirischen Sozialforschung. München: Oldenbourg.

Schwarz, Elke. 2014. @ hannah_arendt. An Arendtian critique of online social networks. Millennium: Journal of International Studies 43:165-186.

Seemann, Michael. 2019. Eine beunruhigende Frage an den digitalen Kapitalismus. APuZ. Aus Politik und Zeitgeschichte 69:10-15.

Spittler, Gerd. 1980. Abstraktes Wissen als Herrschaftsbasis: zur Entstehungsgeschichte bürokratischer Herrschaft im Bauernstaat Preußen. Kölner Zeitschrift für Soziologie und Sozialpsychologie 32:574-604.

Staab, Philipp, und Oliver Nachtwey. 2016. Market and labour control in digital capitalism. triple C: Communication, Capitalism \& Critique 14:457-474.

Stahl, Titus. 2016. Indiscriminate mass surveillance and the public sphere. Ethics and Information Technology 18:33-39.

Stalder, Felix. 2017. Kultur der Digitalität. Berlin: Suhrkamp.

Stark, Birgit. 2019. Mythos „Filterblase“ - Fiktion oder Realität? Der Stand der Forschung aus kommunikationswissenschaftlicher Perspektive. MedienWirtschaft 16:6-10.

Stengel, Richard. 2019. Information wars. How we lost the global battle against disinformation and what we can do about it. New York: Grove Press.

Straßenberger, Grit. 2005. Über das Narrative in der politischen Theorie. Berlin: Akademie Verlag.

Sunstein, Cass R. 2001. Republic.com. Princeton: Princeton University Press.

Sunstein, Cass R. 2009. Republic.com 2.0. Princeton: Princeton University Press.

Süssenguth, Florian (Hrsg.). 2015a. Die Gesellschaft der Daten. Über die digitale Transformation der sozialen Ordnung. Bielefeld: transcript.

Süssenguth, Florian. 2015b. Die Organisation des digitalen Wandels. Zur Funktion von Digitalisierungssemantiken in Wirtschaft, Medien und Politik. In Die Gesellschaft der Daten. Über die digitale Transformation der sozialen Ordnung, Hrsg. Florian Süssenguth, 93-121. Bielefeld: transcript.

Terranova, Tiziana. 2004. Network culture. Politics for the information age. London, Ann Arbor: Pluto Press.

Thiel, Thorsten. 2017. Digitalisierung als Kontext politischen Handelns. Republikanische Perspektiven auf die digitale Transformation der Gegenwart. In Politische Theorie und Digitalisierung, Hrsg. Daniel Jacob, Thorsten Thiel, 189-216. Baden-Baden: Nomos.

Turner, Fred. 2009. Burning man at google. A cultural infrastructure for new media production. New Media \& Society 11:73-94. 
Uricchio, William. 2011. The algorithmic turn. Photosynth, augmented reality and the changing implications of the image. Visual Studies 26:25-35.

von Linder, Jakob. 2020. Wo Zweifler sich in Rage chatten. ZEIT online. https://www.zeit.de/digital/ internet/2020-09/corona-demos-telegram-verschwoerungstheorien-internet-radikalisierung-angsthass. Zugegriffen: 10. Nov. 2020.

Vormbusch, Uwe. 2012. Die Herrschaft der Zahlen. Zur Kalkulation des Sozialen in der kapitalistischen Moderne. Frankfurt a.M., New York: Campus.

Voss, G. Günther, und Cornelia Weiss. 2013. Burnout und Depression - Leiterkrankungen des subjektivierten Kapitalismus oder: Woran leidet der Arbeitskraftunternehmer? In Leistung und Erschöpfung. Burnout in der Wettbewerbsgesellschaft, Hrsg. Sighard Neckel, Greta Wagner, 29-57. Berlin: Suhrkamp.

Wissel, Jan. 2016. Autoritärer Wettbewerbsetatismus. Dynamiken der „marktkonformen Demokratie“ in Europa. In Der Staat des Neoliberalismus, Hrsg. Thomas Biebricher, 263-283. Baden-Baden: Nomos.

Zedner, Lucia. 2007. Pre-crime and post-criminology? Theoretical Criminology 11:261-281.

Zinl, Reinhard. 2016. James Buchanan: Liberaler Gesellschaftsvertrag und freiheitliche Kultur. In Der Staat des Neoliberalismus, Hrsg. Thomas Biebricher, 99-120. Baden-Baden: Nomos.

Zuboff, Shoshana. 2018. Das Zeitalter des Überwachungskapitalismus. Frankfurt, New York: Campus.

Züger, Theresa, Stefania Milan, und Leonie Maria Tanczer. 2017. Sand im Getriebe der Informationsgesellschaft: Wie digitale Technologien die Paradigmen des Zivilen Ungehorsams herausfordern und verändern. In Politische Theorie und Digitalisierung, Hrsg. Daniel Jacob, Thorsten Thiel, 265-296. Baden-Baden: Nomos. 